\title{
Design of Said Weighing Control System Based on Time Error Control
}

\author{
Guangwei Luo and Li Yang \\ Sichuan Engineering Technical College, Electrical and Information Engineering, \\ De Yang, China \\ lgw@scetc.net
}

\begin{abstract}
At present, weighing control system of concrete mixing station mainly uses the discharge valve opening control. And because of unequal drain holes, uneven materials particle sizes, the changing distance between materials and hopper, the material weighing error is large, the concrete mixing ratio error is also imprecise, and the concrete strength quality and curing time have also been great affected. This system uses the time error control principle. According to the material weight error of the last discharge valve open time, the next open time difference is calculated. The last material weight error is compensated. Many discharges effectively solve the error problems of said weighing system and improve measurement accuracy and the mix proportion of concrete. This control method has been successfully applied to the mixing station control system of PLC reconstruction to ensure the system stable operation and concrete curing time, improve weighing precision and concrete strength quality. It has been proven to improve the system running environment and achieve good economic benefits.
\end{abstract}

Keywords: weigh hopper weighing system; PLC; control algorithm; timing control; error

\section{Introduction}

At present, we mainly adopt opening control in concrete mixing control system [1], which is through weighing materiel, to open the open degree of dump valve according to the material weight. The weighing error is impacted by material size, uneven material particle size, moisture content in material and the changes of the distance between the materials and the hopper, and so on, causing larger materials weighing error. If concrete mixing proportion has the larger error, it would impact the quality of concrete strength and solidification time [2]. Entrusted by Zhengheng Company, we carry on the transformation design to the concrete mixing station weighing control system, through checking mixing station, analyzing the factors that impact materials weighing accuracy in the original weighing system, and considering the work environment in the stirring scene at the same time, to control the error through time control principle. The control core is PLC controller with better anti-interference ability, and adopting HMI for remote monitoring, to improve the weighing accuracy of materiel, to improve the concrete mixing proportion, to boost the quality of concrete strength and make sure the concrete time of solidification, and make control system operate simpler, more humane at the same time.

\section{Control System Structure}

Mixing plant control system is mainly to control and monitor the process of material weighing and stir. Its main components are control core PLC, analog input / output 
module, digital I/O module, human-computer interaction HMI and sensor, and so on. The structure of control system is shown in Figure 1.

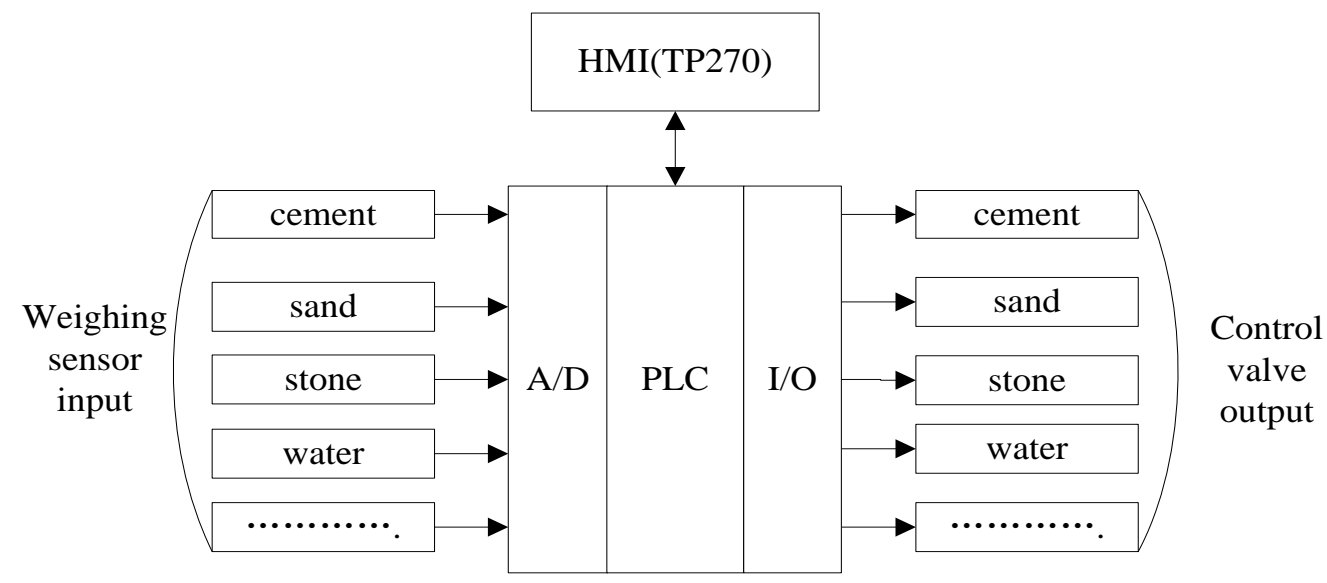

Figure 1. Structure of Control System

PLC as the control core, we select Siemens S7-200PLC, configuring digital and analog extended module and SIWAREX MS weighing function module. It mainly collects, deals with and operates the weight signals of cement, sand, stone, water, coal ash and other materiel, and we adopt Siemens human-computer interface TP270 to set the mixing quantity and the corresponding formula, to revise the operational parameters of control system, and to monitor the current work status at the same time.

\section{Weighing Control Algorithm}

We use hopper scale to detect weight in this design, so we take the weighing process of single material door to establish control algorithm. The relationship between changes of weight and time in weighing process is shown in Figure 2. We use hopper scale to detect weight in this design, so we take the weighing process of single material door to establish control algorithm. The relationship between changes of weight and time in weighing process is shown in Figure 2.

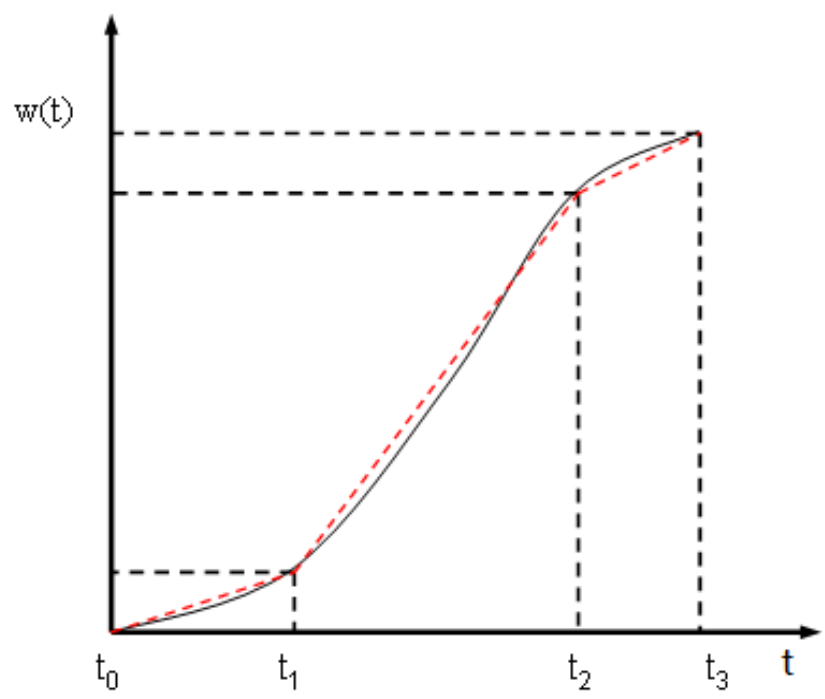

Figure 2. Relationship between Weight Change and Time 
From Figure 2, we could analyze and obtain that the discharge capacity increases with discharge valve opening within per unit time because of discharge valve action process requires execution time within the time of $t_{0}-t_{1}$; the valve is fully open at $t_{1}$, feeding valve is fully open within $t_{1}-t_{2}$; the system starts to execute the close valve command at $t_{2}$, the target meets the requirements and starts to close the door within $t_{2}-t_{3}$, the discharging amount decreases with the discharge valve opening decreases within per unit time.

The time interval is shorter of $t_{0}-t_{1}$ and $t_{2}-t_{3}$ comparing to $t_{1}-t_{2}$, and the feeding quantity is relatively little in feeding amount every time within $t_{0}-t_{1}$ and $t_{2}-t_{3}$. In order to establish mathematical model easily, so we regard the relationship as piecewise linear approximately in Figure 2, and we could obtain the equation of hybrid material weight as follows:

$$
\frac{d w(t)}{d t}=\rho \times v \times k \times s
$$

In the equation, $w(t)$ is the total weight $(\mathrm{kg})$ of hybrid materials; $\rho$ is the density $\left(\mathrm{kg} / \mathrm{m}^{3}\right)$ of hybrid materials; $v$ is the instantaneous velocity $(\mathrm{m} / \mathrm{s})$ of hybrid materials; $s$ is the cross-sectional area $\left(\mathrm{m}^{2}\right)$ of discharge valve; $k$ is the opening coefficient of discharge valve.

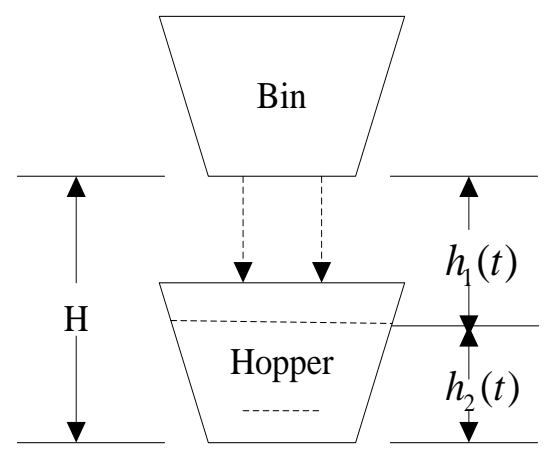

Figure 3. Diagram of Said Weighing

In the process of practical production line, the height of materiel increases gradually in weigh hopper because of hybrid materials fall into the weigh hopper constantly, and it is proportional to the actual weight of material (as shown in Figure 3). We could obtain that:

$$
h_{2}(t)=\alpha w(t)
$$

In the equation, $h_{2}(t)$ is the height (m) of hybrid materials in weigh hopper; $\alpha$ is the proportional coefficient between height and weight of hybrid materials.

If assuming that the distance between discharging door and the bottom of weigh hopper is $\mathrm{H}$, then we could show the distance between discharging door and hybrid material surface $h_{1}(t)$ as:

$$
h_{1}(t)=H-h_{2}(t)=H-\alpha w(t)
$$

The instantaneous velocity of hybrid material fall on the hybrid material surface is:

$$
v=\sqrt{2 g h_{2}(t)}=\sqrt{2 g(H-\alpha w(t))}
$$

Taking equation (4) into equation (1), we could obtain: 


$$
w(t)=\rho s \sqrt{2 g H} t-\rho^{2} s^{2} \alpha t^{2} / 2
$$

Assuming the time of opening material valve is $t_{1}=t+\Delta t$ next time, and then we could obtain:

$$
\left.w\left(t_{1}\right)=\rho s \sqrt{2 g H(t}+\Delta t\right)-\rho^{2} s^{2} \alpha\left(t_{1}+\Delta t\right)^{2} / 2
$$

Then we could obtain the error $w(\Delta t)$ of hybrid material through dealing with above equations:

$$
w(\Delta t)=\rho^{2} s^{2} \alpha g \Delta t t
$$

The relationship among adjustment time $\Delta t$, the time of opening material valve $\mathrm{t}$ and error $w(\Delta t)$ is as follows:

$$
\Delta t=\frac{w(\Delta t)}{\rho^{2} s^{2} \alpha g t}
$$

If the actual weighing error is larger than the set value, the adjustment time moves up $\Delta t$; if the actual weighing error is smaller than the set value, the adjustment time prolongs $\Delta t$. We could reduce the weighing error through adjusting some times.

\section{Practical Application}

We adopt MATLAB to simulate it before practical application, because the coefficients change frequently, the cross-sectional area of discharge valve $s=0.3 \sim 0.1$, the proportional coefficient between height and weight of hybrid materials $\alpha=0.002 \sim 0.001$, the density of hybrid materials $\rho=1000 \sim 10000$, the distance between discharging door and the bottom of weigh hopper $\mathrm{H}=1.2 \sim 1.8$, the free fall acceleration $g=9.8$, we simulate it according to these parameters. The result is shown in Figure 4.

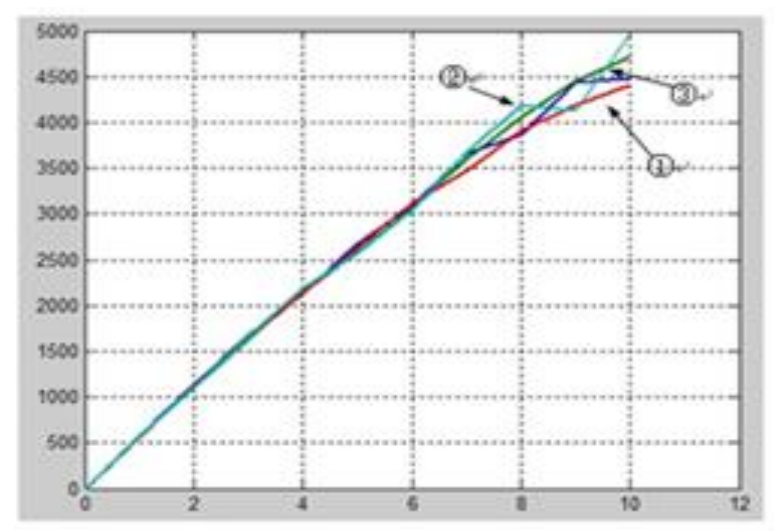

Figure 4. Simulation Effect Diagram

From the result of simulation, putting the weight of an ingredient in multiple weighing, we adjust material valve open time according to the previous weighing error. If the actual weight is less than the set weight in curve (1), we could obtain curve (2) after increasing the valve opening time; and we could obtain curve (3) according to the error in this adjustment valve open time. We carry on iterative calculation some times and adjust the 
valve open time constantly, error is smaller and smaller, and it would meet the actual requirements.

We compare the 5T stone weighing in concrete after reforming the control system, the result is shown in Table 1.

Table 1. Weighing Data Comparison before and after Modification

\begin{tabular}{|c|c|c|c|c|c|c|}
\hline & 1 & 2 & 3 & 4 & 5 & Total \\
\hline $\begin{array}{c}\text { Before } \\
\text { modification }\end{array}$ & 950 & 980 & 990 & 1005 & 970 & 4895 \\
\hline After modification & 980 & 1010 & 998 & 1002 & 995 & 4985 \\
\hline
\end{tabular}

Through the weighing data analysis, system weighing error is about $2 \%$ before modification; system weighing error is just about $0.3 \%$ after modification. So the system improves the accuracy of the system weighing, reduces the weighing error of the material, improves the concrete mix proportion and improves the quality of concrete strength.

\section{Conclusions}

The control system aims at the problems of larger discharge valve opening control of materials weighing error and the influence of concrete strength quality and the solidification time, we put forward the principle of time error control and combine with PLC and HMI at the same time, we successfully resolve the weighing error problems that caused by said weighing material changes in the size of the port system, uneven material particle size and the changes of the distance between the materials and the hopper, improving the measurement precision of weighing system, improving the concrete mix proportion, improving the quality of the concrete and solving the anti-jamming problem of the system. The control system is applied into a stirring device control system in Zhengheng Company successfully, the system runs stable and reliable, materials weighing precision is increased by nearly two percent, improving the quality of the concrete strength, ensuring the coagulation time, improving the operation environment of the system at the same time and achieving good economic benefits.

\section{References}

[1] S. Hong and A. H. Bin, "A Method to Select Radar Signal Based on Blind Source Separation", Modern Radar, vol. 23, no. 3, (2006), pp. 47-50.

[2] X. W. Shu, Z. X. Gan and D. S. dan, "Blind Separation of Radar Signals", Journal of Nanjing University, vol. 42, no. 1, (2006), pp. 38-43.

[3] Q. C. Zhou, H. T. Gao, F. Wang and S. Jie, "A Robust DOA Estimation Algorithm Under the Condition of Unknown Number of Signal Sources", Journal of South China University of Technology (Natural Science Edition), vol. 40, no. 6, (2012), pp. 90-96.

[4] F. Wang and X. S. He, "Underdetermined Blind Separation Based on Ant Colony Clustering", Computer Engineering and Applications, vol. 49, no. 13, (2013), pp. 211-215.

[5] M. K. Pakhira, S. Bandyopadhyay and U. Maulik, "Validity Index for Crisp and Fuzzy Clusters", Pattern Recognition, vol. 37, (2004), pp. 487-501.

[6] S. Cotter, B. Rao and K. Engan, "Sparse Solutions to Linear Inverse Problems With Multiple Measurement Vectors", IEEE Transactions on Signal Processing, vol. 53, no. 7, (2005), pp. 2477-2488.

[7] F. Wang and X. S. He, "Underdetermined Blind Sparation Based on Ant Colony Clustering", Computer Engineering and Applications, vol. 49, no. 13, (2013), pp. 211-215.

[8] A. Cadzow, S. Kim and C. Shiue, "General Direction-of-Arrival Estimation: A Signal Subspace Approach", IEEE Transactions on Aerospace and Electronic Systems, vol. 25, no. 1, (1989), pp. 31-47.

[9] L. De Lathauwer and J. Castaing, "Blind Identification of Underdetermined Mixtures by Simultaneous Matrix Diagonalization”, IEEE Transactions on Signal Processing. vol. 56, no. 3, (2008), pp. 1096-1105.

[10] B. H. Tan, M. Zhao and S. L. Xie, "Blind Separation Algorithms of BPSK Signals by Estimating Source Number", Journal of Electronics \& Information Technology, vol. 31, no. 7, (2009), pp. 1624-1629.

[11] H. Tang and S. Wang, "Parameters Estimation of DSSS Based on Denoising Source Separation", Systems Engineering and Electronics. vol. 33, no. 8, (2011), pp. 1722-1726. 
[12] S. Hosseini, C. Jutten and D. T. Pham, "Markovian Source Separation”, IEEE Transactions on Signal Processing, vol. 51, no. 12, (2003), pp. 3009-3019.

[13] K. Nordhausen, E. Ollila and H. Oja, "On the Performance Indices of ICA and Blind Source Separation", IEEE 12th International Workshop on Signal Processing Advances in Wireless Communications. (2011), pp. 461-465.

[14] Y. J. Shen, G. M. Zhang and S. P. Yang, "New Method for Blind Source Separation in UnderDetermined Mixtures Based on Gabor Transform", Journal of Vibration, Measurement \& Diagnosis, vol. 31, no. 3, (2011), pp. 309-313.

[15] R. J. Wang, Y. J. Han and H. F. Zhou, "Application of Articial Bee Colony Optimization Algorithm in Complex Blind Separation Source", Science China, vol. 44, no. 2, (2014), pp. 199-220.

[16] H. Li and Y. L. Sun, "Under-Determined ICA Algorithm Based on Singular Value Decomposition", Computer Applications and Software, vol. 25, no. 2, (2008), pp. 231-233.

[17] S. Z. Xu, Y. Su and Z. F. Ye, "Blind Source Separation in Underdetermined Mixtures", Journal of Data Acquisition \& Processing, vol. 21, no. 2, (2006), pp. 128-132.

[18] J. Y. Wu, W. J. Li, J. P. Huang, J. L. Zhang and D. R. Chen, "Key techniques for Mobile Internet: a survey, Scientia Sinica Informationis, vol. 45, no. 1, (2015), pp. 45-69. 